\title{
Maternal psychological distress and fetal growth trajectories: The Generation R Study
}

\author{
J. Henrichs ${ }^{1}$, J. J. Schenk ${ }^{1}$, S. J. Roza ${ }^{2}$, M. P. van den Berg ${ }^{2}$, H. G. Schmidt ${ }^{1}$, E. A. P. Steegers ${ }^{3}$, \\ A. Hofman ${ }^{4}$, V. W. V. Jaddoe ${ }^{4,5,6}$, F. C. Verhulst ${ }^{2}$ and H. Tiemeier ${ }^{2 *}$ \\ ${ }^{1}$ Institute of Psychology, Erasmus University Rotterdam, The Netherlands \\ ${ }^{2}$ Department of Child and Adolescent Psychiatry, Erasmus MC - Sophia Children's Hospital, Rotterdam, The Netherlands \\ ${ }^{3}$ Department of Obstetrics and Gynaecology, Erasmus MC - Sophia Children's Hospital, Rotterdam, The Netherlands \\ ${ }^{4}$ Department of Epidemiology and Biostatistics, Erasmus MC University Medical Centre, Rotterdam, The Netherlands \\ ${ }^{5}$ The Generation R Study Group, Erasmus MC University Medical Centre, Rotterdam, The Netherlands \\ ${ }^{6}$ Department of Paediatrics, Erasmus MC - Sophia Children's Hospital, Rotterdam, The Netherlands
}

Background. Previous research suggests, though not consistently, that maternal psychological distress during pregnancy leads to adverse birth outcomes. We investigated whether maternal psychological distress affects fetal growth during the period of mid-pregnancy until birth.

\begin{abstract}
Method. Pregnant women $(n=6313)$ reported levels of psychological distress using the Brief Symptom Inventory (anxious and depressive symptoms) and the Family Assessment Device (family stress) at 20.6 weeks pregnancy and had fetal ultrasound measurements in mid- and late pregnancy. Estimated fetal weight was calculated using head circumference, abdominal circumference and femur length.

Results. In mid-pregnancy, maternal distress was not linked to fetal size. In late pregnancy, however, anxious symptoms were related to fetal size after controlling for potential confounders. Anxious symptoms were also associated with a $37.73 \mathrm{~g}$ [95\% confidence interval (CI) -69.22 to $-6.25, p=0.019]$ lower birth weight. When we related maternal distress to fetal growth curves using multilevel models, more consistent results emerged. Maternal symptoms of anxiety or depression were associated with impaired fetal weight gain and impaired fetal head and abdominal growth. For example, depressive symptoms reduced fetal weight gain by $2.86 \mathrm{~g}$ ( $95 \% \mathrm{CI}-4.48$ to -1.23 , $p<0.001)$ per week.

Conclusions. The study suggests that, starting in mid-pregnancy, fetal growth can be affected by different aspects of maternal distress. In particular, children of prenatally anxious mothers seem to display impaired fetal growth patterns during pregnancy. Future work should address the biological mechanisms underlying the association of maternal distress with fetal development and focus on the effects of reducing psychological distress in pregnancy.
\end{abstract}

Received 6 May 2008; Revised 26 June 2009; Accepted 30 June 2009; First published online 6 August 2009

Key words: Birth cohort, birth weight, fetal growth, maternal psychological distress.

\section{Introduction}

The belief that the emotional state of the pregnant woman affects the development of the fetus is ancient and found in all cultures (Ferreira, 1965). Animal research shows that exposure to prenatal stress is related to lower fetal and birth weight of the offspring (Pinto \& Shetty, 1995; Lesage et al. 2004). In humans, maternal prenatal depression, anxiety and stress are associated with higher rates of spontaneous abortion and pre-eclampsia (Kurki et al. 2000; Nakano et al.

\footnotetext{
* Address for correspondence: H. Tiemeier, Ph.D., Department of Child and Adolescent Psychiatry, Erasmus MC - Sophia Children's Hospital, PO Box 2060, 3000 CB Rotterdam, The Netherlands. (Email: h.tiemeier@erasmusmc.nl)
}

2004). Moreover, maternal psychological distress in pregnancy is related to an increased risk of preterm delivery (Hedegaard et al. 1993; Rondo et al. 2003; Mancuso et al. 2004). Earlier research investigating the relation between maternal psychological distress and lower birth weight was inconsistent. Although some studies reported that maternal psychological distress is negatively related to birth weight (Lou et al. 1994; Rondo et al. 2003; Rahman et al. 2007), other studies observed no (independent) relation between maternal psychological distress and low birth weight (Nordentoft et al. 1996; Andersson et al. 2004; Evans et al. 2007).

Previous studies have investigated the influences of maternal prenatal distress on birth outcomes, such as birth weight. Birth outcomes are only crude summary 
Table 1. Listing of items included in the depression and anxiety scale of the Brief Symptom Inventory

\begin{tabular}{|c|c|c|c|c|c|}
\hline \multicolumn{6}{|l|}{ Depression scale } \\
\hline \multicolumn{6}{|c|}{ During the past 7 days, how much were you distressed by: } \\
\hline 1. Thoughts of ending your life & 0 Not at all & 1 A little bit & 2 Moderately & 3 Quite a bit & 4 Extremely \\
\hline 2. Feeling lonely & 0 Not at all & 1 A little bit & 2 Moderately & 3 Quite a bit & 4 Extremely \\
\hline 3. Feeling blue & 0 Not at all & 1 A little bit & 2 Moderately & 3 Quite a bit & 4 Extremely \\
\hline 4. Feeling no interest in things & 0 Not at all & 1 A little bit & 2 Moderately & 3 Quite a bit & 4 Extremely \\
\hline 5. Feeling hopeless about the future & 0 Not at all & 1 A little bit & 2 Moderately & 3 Quite a bit & 4 Extremely \\
\hline 6. Feelings of worthlessness & 0 Not at all & 1 A little bit & 2 Moderately & 3 Quite a bit & 4 Extremely \\
\hline \multicolumn{6}{|l|}{ Anxiety scale } \\
\hline \multicolumn{6}{|c|}{ During the past 7 days, how much were you distressed by: } \\
\hline 1. Nervousness or shaking inside & 0 Not at all & 1 A little bit & 2 Moderately & 3 Quite a bit & 4 Extremely \\
\hline 2. Suddenly scared for no reason & 0 Not at all & 1 A little bit & 2 Moderately & 3 Quite a bit & 4 Extremely \\
\hline 3. Feeling fearful & 0 Not at all & 1 A little bit & 2 Moderately & 3 Quite a bit & 4 Extremely \\
\hline 4. Feeling tense or keyed up & 0 Not at all & 1 A little bit & 2 Moderately & 3 Quite a bit & 4 Extremely \\
\hline 5. Spells of terror or panic & 0 Not at all & 1 A little bit & 2 Moderately & 3 Quite a bit & 4 Extremely \\
\hline 6. Feeling so restless you couldn't sit still & 0 Not at all & 1 A little bit & 2 Moderately & 3 Quite a bit & 4 Extremely \\
\hline
\end{tabular}

measures of intrauterine growth and cannot provide information on the growth of the fetal head, abdomen, femur and body across different time periods in pregnancy. Furthermore, individuals can reach the same birth weight by different fetal growth trajectories (Bloomfield et al. 2006). Therefore, in the current population-based cohort study, we examined the effect of maternal distress during pregnancy not only on birth weight but also on repeatedly measured fetal growth parameters such as head and abdominal circumference and femur length in mid- and late pregnancy. Furthermore, we also studied the ratio of abdominal and head circumference, which assesses levels of symmetry of fetal growth and is an indicator of brain sparing. We hypothesized that maternal distress in pregnancy negatively affects fetal size and growth from mid-pregnancy onwards.

\section{Method}

\section{Design}

This study was embedded in the Generation R Study, a population-based cohort study from fetal life onwards in Rotterdam, The Netherlands. The Generation $\mathrm{R}$ Study has previously been described in detail (Jaddoe et al. 2006). The cohort includes 9778 mothers and their children that were born between April 2002 and January 2006. Assessments in pregnant women consisted of physical examinations, fetal ultrasounds, biological samples and questionnaires.

The study has been approved by the Medical Ethics Committee of the Erasmus Medical Centre, Rotterdam (no. MEC 198.782/2001/31). Written informed consent was obtained from all pregnant women.

\section{Population for analysis}

Of the total cohort of 9778 mothers, 8880 (91\%) were enrolled in pregnancy (Jaddoe et al. 2006). In this study, 104 fetal deaths and 93 mothers with twin pregnancies were excluded because growth potentials of fetuses in multiple pregnancies are not comparable with those of fetuses in singleton pregnancies. For mothers with multiple pregnancies, data on their second $(n=500)$ or third $(n=8)$ pregnancy enrolled in the study were excluded to avoid effects of paired data. The remaining 8130 mothers were eligible. There were 45 losses to follow-up during pregnancy. In $22.2 \% \quad(n=1806)$ of the eligible mothers, no information on any of the three types of maternal distress was available. For 11 mothers there were no data on fetal ultrasound. Of the remaining 6313 (77.7\%) mothers, 5976 mothers $(94.7 \%)$ had two ultrasound assessments in mid- and late pregnancy and 337 (5.3\%) mothers attended only one ultrasound assessment.

\section{Maternal psychological distress in pregnancy}

Information on maternal distress was obtained by postal questionnaires that were returned at, on average, 20.6 (s.D. =1.2) weeks of gestation. Anxious and depressive symptoms were assessed with the Brief Symptom Inventory (BSI), a validated self-report questionnaire with 53 items (De Beurs, 2004). These items define a spectrum of psychiatric symptoms in the preceding 7 days. For this study, the six-item anxiety scale and the six-item depression scale were used (Table 1). Each item was rated on five-point unidimensional scales ranging from ' 0 ' (not at all) to ' 4 ' (extremely). Total scores for each scale were calculated 
by summing the item scores (range: $0-4$ ) and dividing by the number of endorsed items. Following the BSI manual instructions (De Beurs, 2004) we allowed one missing item per scale to minimize selective nonresponse. For depressive symptoms, $1.6 \%(n=100)$ of the participating mothers only filled in five of the six items. For anxious symptoms, one item was missing in $1.9 \%(n=120)$ of the mothers. The internal consistencies were $\alpha=0.80$ for the depression scale and $\alpha=0.75$ for the anxiety scale. Mothers scoring in the top $15 \%$ of the anxiety or depression scale scores of the BSI were considered to have anxious or depressive symptoms. The applied top $15 \%$ cut-offs were 0.50 for depressive symptoms and 0.66 for anxious symptoms, and lie within the range used to describe 'above average' scores, i.e. scores $>0.33$ and $<0.67$, on both the depression and the anxiety scale of the BSI in the Dutch norm population (De Beurs, 2004). An earlier study used a very similar percentile cut-off to define increased antenatal anxiety using the Crown-Crisp Index (Birtchnell et al. 1988; O'Connor et al. 2002).

Within a Generation R subgroup of 917 women, we tested the BSI's ability to identify clinical depression and anxiety using the applied cut-off scores. Data on clinical depression and anxiety during the last year were obtained with the Composite International Diagnostic Interview (CIDI). The CIDI is a structured interview based on DSM-IV criteria. Good reliability and validity have been reported (Andrews \& Peters, 1998). A home interview was conducted during pregnancy by research assistants. The cut-offs for each scale had low positive predictive values for depressive $(6.8 \%)$ and anxious $(10.4 \%)$ disorders, but they were very good at assessing that a person is not depressed or anxious (negative predictive value $=99.2 \%$ or negative predictive value $=99.3 \%$, respectively). However, if the prevalence is as low as in this subgroup, i.e. $<2 \%$ for clinical depression and anxiety, the positive predictive value will not be close to 1 even if sensitivity and specificity are high. Inevitably most people with positive test results will be false positives (Altman \& Bland, 1994). Therefore, we also calculated the positive likelihood ratio $(\mathrm{LR}+)$ of the top $15 \%$ cutoffs for depressive $(\mathrm{LR}+=5.62)$ and anxious symptoms $(\mathrm{LR}+=9.68)$, which accounts for the prevalence. This demonstrated moderate quality of the cut-offs as indicators of certainty of diagnosis.

Family stress was assessed by the 7th subscale General Functioning (GF) of the Family Assessment Device (Byles et al. 1988). GF is a validated 12-item measure of family health. The item scores were summed and divided by 12 , yielding a total score from 1 to 4 . We allowed $25 \%$ of the 12 GF items to be missing, which was the case in $4.2 \%(n=263)$ of the participating mothers in whom weighted sum-scores were calculated. A GF score $>2.17$ (cut-off) denotes unhealthy family functioning. In this study, just as in the Ontario Child Health Study, 10\% of the families scored above this cut-off (Byles et al. 1988). The internal consistency of GF was $\alpha=0.90$.

\section{Fetal ultrasound measurements and birth weight}

Trained sonographers conducted fetal ultrasound examinations at the visits to the research centres in early (gestational age $<18$ weeks), mid-pregnancy (gestational age 18-25 weeks) and late pregnancy (gestational age $\geqslant 25$ weeks). These examinations were used for establishing gestational age and assessing fetal growth characteristics. Gestational age was established by the fetal ultrasound assessments since women do not remember the exact date of their last menstrual period or have irregular menstrual cycles (Altman \& Chitty, 1997).

Online measurements included head and abdominal circumference, and femur length in mid- and late pregnancy that were all measured to the nearest millimetre using standardized techniques. The intraand inter-observer reliability of fetal biometry in early pregnancy within the Generation R Study was high. Intra-observer intraclass correlation coefficients based on relative agreement varied from 0.982 to 0.995 , interobserver intraclass correlation coefficients varied from 0.982 to 0.988 , with coefficients of variation between $2.2 \%$ and $5.9 \%$ (Verburg et al. 2008a). The ratio of abdominal and head circumference, which was calculated by dividing abdominal circumference through head circumference, measures symmetry of fetal growth and indicates brain sparing. Estimated fetal weight was calculated using the formula by Hadlock et al. (1984) including head and abdominal circumference, and femur length. This formula by Hadlock et al. (1984) is frequently used in research and applied within Dutch medical practice. Before 18 weeks of gestation an accurate estimation of fetal weight cannot be achieved (Hadlock et al. 1984). Gestational ageadjusted standard deviation scores of estimated fetal weight were constructed using reference growth curves from the total Generation R Study population (Verburg et al. 2008b). Birth weight was obtained from medical records completed by midwives and gynaecologists.

\section{Covariates}

Information on maternal age, pre-pregnancy body mass index, educational level, ethnicity and parity $(0$, or $\geqslant 1)$ was obtained by questionnaire at enrolment. Following the definition of Statistics Netherlands we divided education into five categories: primary education (no education, primary school), secondary 
education 1st phase (lower vocational training or $\leqslant 3$ years secondary school), secondary education 2nd phase ( $>3$ years secondary school, intermediate vocational training), higher education 1st phase (higher vocational training) and higher education 2nd phase (university degree). Ethnicity of the mother was based on the country of birth of herself and her parents. Maternal height was measured during the first visit to the research centre. Information about maternal prenatal smoking and alcohol use was obtained by questionnaires in early, mid- and late pregnancy. Based on these questionnaires maternal smoking or alcohol use were categorized into 'no', 'until pregnancy was known' and 'continued during pregnancy' as described previously (Roza et al. 2007). Fetal sex and information on gestational diabetes, pre-eclampsia, and maternal hypertension during pregnancy were obtained from medical records.

\section{Statistical analysis}

To examine whether non-response was selective, we compared core data of pregnant women with information on psychological distress and fetal ultrasound assessments with eligible women not included because of missing data on one or the other assessment.

Multiple linear regression was used to examine the associations of maternal distress with absolute measures of fetal size in mid- and late pregnancy and birth weight. To investigate whether the wide range of gestational ages, in which fetal size was assessed, influenced our results we reran analysis using gestational age-adjusted standard deviation scores of estimated fetal weight as outcome measures. All models were controlled for maternal education and known determinants of fetal development, i.e. maternal height, age, body mass index, ethnicity, prenatal smoking, parity, gestational diabetes, pre-eclampsia, hypertension and fetal sex (Kramer, 1987). Models including absolute measures of fetal size were additionally controlled for gestational age. Furthermore, all analyses were also adjusted for maternal anxious symptoms or for family stress to determine whether a type of maternal distress was independently related to fetal size. To avoid collinearity and over-adjustment, maternal anxious and depressive symptoms were not included in the same model. Anxiety and depression as measured by the BSI were highly co-morbid (correlation: $r=0.7, p<0.001$ ). Maternal prenatal alcohol use did not significantly improve the models and was therefore not included in the analysis. On average, data were incomplete in 3.5\% (range: $0.0-15.7 \%$ ) of the confounders. To avoid the bias of a complete case analysis we accounted for missing information on confounders by using a missing dum- my category for categorical variables or imputing the mean or median. The number of missing data per covariate is shown in Table 2. Using a categorical distinction of a top $15 \%$ cut-off for anxious and depressive symptoms and the established cut-off of a GF score $>2.17$ for family stress (Byles et al. 1988), we established dichotomized main determinants that were used in our primary analyses.

The associations of maternal distress with repeatedly measured parameters of fetal growth were analysed using longitudinal multilevel analysis to account for the dependency between measurements in the same subject. As fetal growth trajectories follow a non-linear pattern we used fractional polynomials of gestational age to model fetal growth. Fractional polynomials account for non-linearity and offer greater flexibility in curve shape than conventional polynomials (Royston \& Altman, 1994; Royston et al. 1999). We fitted an additive regression model to estimate fetal growth; the resulting variable was a sum of transformations of gestational age. A fractional polynomial of second-degree in a variable $x$ (in this case gestational age) is a linear combination of power transformations of the form: $\beta_{0}+\beta_{1} \mathrm{x}^{\mathrm{p} 1}+\beta_{2} \mathrm{x}^{\mathrm{p} 2}$. The transformation functions were chosen from first- or second-degree powers among from $\mathrm{P}=(-2,-1$, $-0.5,0,0.5,1,2,3)$. The best-fitting model was chosen by comparing the deviance difference of the respective fractional polynomial regression model with the straight line model using approximate $\chi^{2}$ tests with significance level set at 0.1 (Royston et al. 1999). Random effects for both intercept and gestational age were included. Then, type of maternal distress was brought into the model as the main determinant. The interaction term of maternal distress with gestational age was included in the model to compare the slope of the curves between the different categories of affective symptoms and family stress. We tested whether this interaction term resulted in a significant improvement by comparing the $-2 \log$ likelihood of the model with the interaction term with the $-2 \log$ likelihood of the model without the interaction term. The following models were used:

Head circumference $=\beta_{0}+\left(\beta_{1} \times\right.$ maternal distress $)+$ $\left(\beta_{2} \times\right.$ gestational age $)+\left(\beta_{3} \times\right.$ gestational age $\left.{ }^{2}\right)+\left[\beta_{4} \times\right.$ gestational age $\mathrm{e}^{2} \times \ln ($ gestational age $\left.)\right]+\left(\beta_{5} \times\right.$ maternal distress $\times$ gestational age).

Ratio of abdominal and head circumference $=$ $\beta_{0}+\left(\beta_{1} \times\right.$ maternal distress $)+\left(\beta_{2} \times\right.$ gestational age $)+$ $\left[\beta_{3} \times \ln (\right.$ gestational age $\left.)\right]+\left(\beta_{4} \times\right.$ gestational age a. $\left.^{-0.5}\right)+$ $\left(\beta_{5} \times\right.$ maternal distress $\times$ gestational age $)$.

Femur length $=\beta_{0}+\left(\beta_{1} \times\right.$ maternal distress $)+\left(\beta_{2} \times\right.$ gestational age $)+\left(\beta_{3} \times\right.$ gestational age $\left.\mathrm{e}^{3}\right)+\left(\beta_{4} \times\right.$ maternal distress $\times$ gestational age). 
Table 2. Maternal and child characteristics by level of depressive symptoms ${ }^{\mathrm{a}}$

\begin{tabular}{|c|c|c|}
\hline & $\begin{array}{l}\text { No depressive } \\
\text { symptoms }(n=5372)\end{array}$ & $\begin{array}{l}\text { Depressive } \\
\text { symptoms }(n=941)\end{array}$ \\
\hline \multicolumn{3}{|l|}{ Maternal characteristics } \\
\hline Age, years & $30.1(5.0)$ & $27.9(5.7)^{* * *}$ \\
\hline Height, $\mathrm{cm}$ & $167.8(7.3)$ & $165.2(7.2)^{* * *}$ \\
\hline \multicolumn{3}{|l|}{ Pre-pregnancy body mass index, $\mathrm{kg} / \mathrm{m}^{2}$} \\
\hline Median (95\% range) & $22.5(18.0-34.6)$ & $22.8(17.6-35.6)$ \\
\hline Parity, \% nulliparous & 37.8 & 41.1 \\
\hline \multicolumn{3}{|l|}{ Education, \% } \\
\hline Primary education & 8.0 & 18.6 \\
\hline Secondary education 1 st phase & 14.2 & 23.9 \\
\hline Secondary education 2 nd phase & 29.9 & 37.7 \\
\hline Higher education 1st phase & 21.6 & 12.1 \\
\hline Higher education 2nd phase & 26.3 & $7.8^{* * *}$ \\
\hline \multicolumn{3}{|l|}{ Ethnicity, \% } \\
\hline Dutch & 57.4 & 25.2 \\
\hline Cape Verdian & 3.1 & 8.2 \\
\hline Moroccan & 4.7 & 10.2 \\
\hline Dutch Antilles & 2.9 & 6.1 \\
\hline Surinamese & 7.8 & 14.5 \\
\hline Turkish & 6.7 & 17.9 \\
\hline Other Western & 12.3 & 9.8 \\
\hline Other non-Western & 5.1 & $8.2^{* * *}$ \\
\hline \multicolumn{3}{|l|}{ Smoking during pregnancy, \% } \\
\hline No & 76.9 & 62.5 \\
\hline Until pregnancy was known & 7.6 & 6.9 \\
\hline Continued during pregnancy & 15.5 & $30.6^{* * *}$ \\
\hline \multicolumn{3}{|l|}{ Alcohol use in pregnancy, \% } \\
\hline No & 43.2 & 53.9 \\
\hline Until pregnancy was known & 13.4 & 10.8 \\
\hline Continued during pregnancy & 43.3 & $35.3^{* * *}$ \\
\hline Gestational diabetes, \% yes & 1.1 & 0.7 \\
\hline Pre-eclampsia, \% yes & 1.8 & 2.2 \\
\hline Hypertension, \% yes & 4.3 & 2.9 \\
\hline \multicolumn{3}{|l|}{ Child characteristics } \\
\hline Sex, \% girls & 50.7 & 47.5 \\
\hline Gestational age in mid-pregnancy, weeks & $20.6(1.1)$ & $20.7(1.3)$ \\
\hline Head circumference in mid-pregnancy, $\mathrm{mm}$ & $179.3(14.2)$ & $179.7(15.6)$ \\
\hline Abdominal circumference in mid-pregnancy, $\mathrm{mm}$ & $156.6(14.6)$ & $156.9(15.4)$ \\
\hline Femur length in mid-pregnancy, mm & $33.4(3.5)$ & $33.6(3.8)$ \\
\hline Estimated fetal weight in mid-pregnancy, $\mathrm{g}$ & $380.9(91.9)$ & $383.2(97.5)$ \\
\hline Gestational age in late pregnancy, weeks & $30.4(1.1)$ & $30.4(1.1)$ \\
\hline Head circumference in late pregnancy, $\mathrm{mm}$ & $285.1(12.3)$ & $283.5(12.5)^{* * *}$ \\
\hline Abdominal circumference in late pregnancy, $\mathrm{mm}$ & $264.1(16.2)$ & $261.8(17.3)^{* * *}$ \\
\hline Femur length in late pregnancy, $\mathrm{mm}$ & $57.4(2.9)$ & $57.3(3.0)$ \\
\hline Estimated fetal weight in late pregnancy, $g$ & $1618(251)$ & $1592(265)^{* *}$ \\
\hline Birth weight, $g$ & $3431(554)$ & $3347(552)^{* * *}$ \\
\hline Gestational age at birth, weeks & $39.9(1.7)$ & $39.8(1.8)$ \\
\hline
\end{tabular}

Values are given as mean (standard deviation) unless otherwise indicated.

${ }^{a}$ Independent $t$ tests were used for continuous normal distributed variables, $\chi^{2}$ tests were used for categorical variables and Mann-Whitney $U$ tests for continuous non-normal distributed variables. Data were missing on height $(n=16)$, pre-pregnancy body mass index $(n=989)$, parity $(n=37)$, education $(n=319)$, ethnicity $(n=232)$, alcohol use during pregnancy $(n=380)$, gestational diabetes $(n=225)$, pre-eclampsia $(n=225)$ and hypertension during pregnancy $(n=224)$.

${ }^{*} p<0.05, * * p<0.01,{ }^{* * *} p<0.001$. 
Table 3. Associations of maternal distress during pregnancy with fetal size in mid-and late pregnancy and size at birth ${ }^{\mathrm{a}}$

\begin{tabular}{llll}
\hline $\begin{array}{l}\text { Type of } \\
\text { maternal distress }\end{array}$ & $\begin{array}{l}\text { Estimated fetal weight } \\
\text { in mid-pregnancy, } \mathrm{g}^{\mathrm{b}}\end{array}$ & $\begin{array}{l}\text { Estimated fetal weight } \\
\text { in late pregnancy, } \mathrm{g}^{\mathrm{b}}\end{array}$ & Birth weight, $\mathrm{g}^{\mathrm{b}}$ \\
\hline $\begin{array}{l}\text { Depressive symptoms } \\
\text { Anxious symptoms }\end{array}$ & $-0.53(-3.85$ to 2.79$)$ & $-2.09(-15.62$ to 11.44$)$ & $-22.42(-53.05$ to 8.21$)$ \\
Family stress & $-1.94(-5.35$ to 1.47$)$ & $-15.72(-29.57 \text { to }-1.87)^{*}$ & $-37.73(-69.22 \text { to }-6.25)^{*}$ \\
\hline
\end{tabular}

Values are given as $\beta$ coefficient (95\% confidence interval).

a Adjusted for gestational age in mid- or late pregnancy or at birth, fetal sex, maternal age, height, body mass index, education, ethnicity, smoking during pregnancy, parity, gestational diabetes, hypertension in pregnancy, pre-eclampsia and for maternal anxious symptoms in pregnancy in the case of family stress or for family stress in the case of maternal anxious/depressive symptoms.

${ }^{\mathrm{b}} \beta$ Coefficients represent the differences in fetal or birth weight between high levels of maternal distress and low levels of maternal distress.

${ }^{*} p<0.05$.

Fetal weight gain $=\beta_{0}+\left(\beta_{1} \times\right.$ maternal distress $)+\left(\beta_{2} \times\right.$ gestational age $)+\left[\beta_{3} \times\right.$ gestational age $\times \ln ($ gestational age $)]+\left(\beta_{4} \times\right.$ maternal distress $\times$ gestational age $)$.

The model of abdominal circumference was the same as that of head circumference. The model of fetal weight gain represents the increase in weight of the fetus from mid-pregnancy onwards and is based on estimated fetal weight in mid- and late pregnancy and birth weight. In these models, ' $\beta_{0}+\left(\beta_{1} \times\right.$ maternal distress)' reflects the intercept and the terms including ' $\beta_{\mathrm{x}} \times$ gestational age' (or ' $\beta_{\mathrm{x}} \times$ polynomials of gestational age') reflect the slope of fetal growth per week. Terms including ' $\beta_{\mathrm{x}} \times$ maternal distress $\times$ gestational age' represent the differences in growth per week of the respective fetal body part (or in fetal weight gain) between the categories of maternal distress. Models were based on 11856 observations for head circumference, 11915 observations for abdominal circumference, 11570 observations for the ratio of abdominal and head circumference, 11925 observations for femur length and 18010 observations for fetal and birth weight. All models were controlled for potential confounders. Then, all models were additionally adjusted for maternal anxious symptoms or for family stress as well as for the respective interaction with gestational age to determine whether a type of maternal distress was independently related to fetal growth trajectories. SPSS for Windows version 15.0 (SPSS Inc., USA) and SAS version 9.1 (SAS Institute Inc., USA) including the Proc Mixed module for longitudinal multilevel analysis were used.

\section{Non-response analysis}

The non-response analysis showed that mothers included in the study were more likely to be Dutch $\left[52.6 \%\right.$ v. $\left.30.6 \%, \chi^{2}(7)=327.9, p<0.001\right]$ and to be higher educated [higher education with a university degree $23.7 \%$ v. $13.3 \%, \chi^{2}(4)=266.3, p<0.001$ ] than non-responders. Children of mothers in the study had a higher birth weight [3416 (S.D.=556) g v. 3343 (S.D. $=581) \mathrm{g}, t=4.83, p<0.001]$ and gestational age at birth [39.9 (S.D. $=1.8$ ) weeks $v .39 .6$ (S.D. $=2.3$ ) weeks, $t=5.99, p<0.001]$.

\section{Results}

Table 2 presents maternal and child characteristics of mothers with and without depressive symptoms during pregnancy. Mothers reporting depressive symptoms were younger, less tall, had higher rates of education, were less often Dutch, and continued smoking during pregnancy more often than mothers not reporting depressive symptoms. Children of mothers with depressive symptoms during pregnancy had lower fetal weight in late pregnancy and lower birth weight (Table 2). Distributions for mothers who reported anxious symptoms $(n=937)$ or family stress $(n=625)$ and who did not report anxious symptoms $(n=5376)$ or family stress $(n=5688)$ were similar (data not shown).

Family stress in pregnancy was moderately correlated with both anxiety $(r=0.3, p<0.001)$ and depression $(r=0.4, p<0.001)$. Head and abdominal circumference, and femur length were all highly correlated in late pregnancy $(r=0.6, p<0.001)$. Correlations between these ultrasound measurements in mid-pregnancy were similar (data not shown).

Table 3 shows that maternal distress was not related to estimated fetal weight in mid-pregnancy. In contrast, a crude analysis demonstrated that all types of maternal distress were negatively associated with fetal weight in late pregnancy (data not shown). However, only anxious symptoms were negatively linked to 
estimated fetal weight in late pregnancy after controlling for potential confounders (Table 3). Almost identical results were found when we used standard deviation scores of estimated fetal weight as outcome. Anxious symptoms were negatively related to standard deviation scores of estimated fetal weight in late pregnancy $[\beta=-0.09,95 \%$ confidence interval (CI) -0.17 to $-0.02, p=0.013$ ] but not in mid-pregnancy $(\beta=-0.06,95 \% \mathrm{CI}-0.14$ to $0.02, p=0.123)$. The other forms of maternal distress were not related to standard deviation scores of estimated fetal weight in mid- and late pregnancy after adjustment for potential confounders (data not shown). Similarly, all forms of maternal distress were negatively related to birth weight before adjustments were made (data not shown). After controlling for potential confounders, only anxious symptoms were associated with lower birth weight (Table 3).

Table 4 presents the adjusted associations between maternal distress and repeatedly measured fetal growth characteristics. The effect estimates of the different forms of maternal distress, the respective slope based on fractional polynomials of gestational age and the interaction terms of maternal distress with gestational age are shown. The main effects of maternal distress on the fetal growth characteristics cannot be interpreted because the interaction effects were included in the models. Anxious symptoms were negatively associated with growth trajectories of the fetal head and abdomen and with fetal weight gain but not with growth patterns of the femur or asymmetric growth. Depressive symptoms had a negative association with fetal head growth and fetal weight gain but not with growth of the femur, abdomen or asymmetric growth. Family stress was not related to any parameter of fetal growth. However, when the association between family stress and the different fetal growth characteristics was not additionally adjusted for anxious symptoms but only for the other confounders we did find significant associations. Family stress was negatively linked to fetal head growth $(\beta=$ $-0.09 \mathrm{~mm} /$ week, $95 \% \mathrm{CI}-0.16$ to $-0.01, p=0.024)$ and fetal weight gain $(\beta=-2.53 \mathrm{~g} /$ week, $95 \% \mathrm{CI}$ -4.41 to $-0.13, p=0.009)$. To illustrate the non-linear pattern of the modelled fetal growth trajectories, Fig. 1 presents patterns of weight gain of fetuses of mothers with and without anxious symptoms during pregnancy.

To place the magnitude of observed effects on the rate of fetal weight gain we also investigated the association of maternal prenatal smoking with fetal weight gain. Maternal smoking during pregnancy was linked to a $7.33 \mathrm{~g}(95 \% \mathrm{CI}-8.84$ to $-5.82, p<0.001)$ lower fetal weight gain per week after control for potential confounders. In comparison with, for example, the negative effect of maternal anxious symptoms on fetal weight gain (i.e. $\beta=-3.23 \mathrm{~g} /$ week, $95 \% \mathrm{CI}-4.91$ to $-1.55, p=0.002)$, the negative effect of maternal smoking on fetal weight gain was 2.3 times higher.

\section{Discussion}

In this study we showed that affective symptoms during pregnancy were negatively associated with growth trajectories of, in particular, fetal head and abdominal circumference. Furthermore, children of mothers with anxious or depressive symptoms had reduced fetal weight gain during pregnancy. Only maternal anxious symptoms during pregnancy were related to lower birth weight.

So far, studies relating maternal psychological distress to lower birth weight have shown inconsistent findings. While some studies found no (independent) association between maternal psychological distress and low birth weight (Nordentoft et al. 1996; Andersson et al. 2004; Evans et al. 2007), our results as regards maternal anxious symptoms in pregnancy are in line with the positive findings from earlier studies of birth weight (Lou et al. 1994; Rondo et al. 2003; Rahman et al. 2007). However, birth weight is only a summative measure of a long, rapid and non-linear period of intrauterine growth. While undergoing fetal growth restriction due to environmental influences an individual fetus may still reach a normal birth weight because of his/her high genetic growth potential. Nevertheless, fetal growth restriction may affect fetal physiology and lifetime health (Hanson, 2002).

Only a single cross-sectional study reported an association between maternal psychological distress and fetal size in mid-pregnancy, indexed by fetal weight (Diego et al. 2006). This study was based on a small sample $(n=98)$ with measurements in mid-pregnancy only and an incomplete control for confounders.

In our study, maternal distress was related to fetal size in late pregnancy and at birth but not to fetal size in mid-pregnancy, which suggests that influences of maternal distress on fetal growth are strongest in the last trimester of pregnancy. This is not surprising because fetal growth prior to 20 weeks is predominantly determined by genetic predisposition, whereas growth in the third trimester is more likely to be related to intrauterine environment. The finding may also reflect that effects of maternal distress on fetal growth are cumulative and easier to detect in the last trimester of pregnancy because of the increasing discriminative power of the measurements.

Maternal distress was associated with reduced fetal weight gain, and growth of the fetal head and abdomen but not with growth of the femur. Probably, maternal distress affects development of central 
Table 4. Associations of maternal depressive and anxious symptoms and family stress in pregnancy with fetal growth

\begin{tabular}{|c|c|c|c|c|c|}
\hline Type of maternal distress & Head circumference $^{\mathrm{b}}$ & Abdominal circumference ${ }^{b}$ & $\begin{array}{l}\text { Ratio of abdominal and } \\
\text { head circumference }{ }^{b}\end{array}$ & Femur length ${ }^{\mathrm{b}}$ & Fetal weight gain ${ }^{\mathrm{b}}$ \\
\hline No depressive symptoms & Reference & Reference & Reference & Reference & Reference \\
\hline Depressive symptoms & $1.52(0.01-3.04)^{*}$ & $0.90(-1.16$ to 2.96$)$ & $-0.00(-0.01$ to 0.01$)$ & $0.34(-0.04$ to 0.73$)$ & $61.13(24.99-97.27)^{* * * *}$ \\
\hline GA & $-59.01(-67.36 \text { to }-50.66)^{* * *}$ & $-33.61(-45.02 \text { to }-22.19)^{* * *}$ & $0.08(0.04-0.11)^{* * *}$ & $3.35(3.28-3.42)^{* * *}$ & $-752.2(-763.5 \text { to }-740.9)^{* * *}$ \\
\hline $\mathrm{GA}^{2}$ & $7.14(6.35-7.93)^{* * *}$ & $4.33(3.24-5.41)^{* * *}$ & - & - & - \\
\hline $\mathrm{GA}^{3}$ & - & - & - & $-0.00(-0.00 \text { to }-0.00)^{* * * *}$ & - \\
\hline $\mathrm{GA}^{-0.5}$ & - & - & $-30.92(-47.45 \text { to }-14.39)^{* * *}$ & - & - \\
\hline $\ln (\mathrm{GA})$ & - & - & $-4.87(-7.37 \text { to }-2.37)^{* * * *}$ & - & - \\
\hline $\mathrm{GA} \times \ln (\mathrm{GA})$ & - & - & - & - & $207.2(204.6-209.7)^{* * *}$ \\
\hline $\mathrm{GA}^{2} \times \ln (\mathrm{GA})$ & $-1.54(-1.71 \text { to }-1.37)^{* * *}$ & $-0.92(-1.15 \text { to }-0.69)^{* * *}$ & - & - & - \\
\hline $\mathrm{GA} \times$ no depressive symptoms & Reference & Reference & Reference & Reference & Reference \\
\hline $\mathrm{GA} \times$ depressive symptoms & $-0.07(-0.13 \text { to }-0.01)^{*}$ & $-0.06(-0.15$ to 0.03$)$ & $0.00(-0.00$ to 0.00$)$ & $-0.01(-0.02$ to 0.00$)$ & $-2.86(-4.48 \text { to }-1.23)^{* * *}$ \\
\hline No anxious symptoms & Reference & Reference & Reference & Reference & Reference \\
\hline Anxious symptoms & $2.23(0.66-3.80)^{* *}$ & $1.85(-0.28$ to 3.98$)$ & $-0.00(-0.01$ to 0.01$)$ & $0.23(-0.16$ to 0.63$)$ & $66.75(23.37-98.63)^{* *}$ \\
\hline GA & $-58.93(-67.28 \text { to }-50.58)^{* * *}$ & $-33.31(-44.73 \text { to }-21.90)^{* * *}$ & $0.08(0.04-0.11)^{* * *}$ & $3.35(3.28-3.42)^{* * *}$ & $-752.2(-763.5 \text { to }-740.3)^{* * *}$ \\
\hline $\mathrm{GA}^{2}$ & $7.13(6.34-7.92)^{* * * *}$ & $4.30(3.21-5.38)^{* * * *}$ & - & - & - \\
\hline $\mathrm{GA}^{3}$ & - & - & - & $-0.00(-0.00 \text { to }-0.00)^{* * * *}$ & - \\
\hline $\mathrm{GA}^{-0.5}$ & - & - & $-31.30(-47.83 \text { to }-14.77)^{* * *}$ & - & - \\
\hline $\ln (\mathrm{GA})$ & - & - & $-4.93(-7.43 \text { to }-2.42)^{* * *}$ & - & - \\
\hline $\mathrm{GA} \times \ln (\mathrm{GA})$ & - & - & - & - & $207.2(204.6-209.7)^{* * *}$ \\
\hline $\mathrm{GA}^{2} \times \ln (\mathrm{GA})$ & $-1.54(-1.71 \text { to }-1.37)^{* * *}$ & $-0.92(-1.15 \text { to }-0.69)^{* * *}$ & - & - & - \\
\hline $\mathrm{GA} \times$ no anxious symptoms & Reference & Reference & Reference & Reference & Reference \\
\hline $\mathrm{GA} \times$ anxious symptoms & $-0.10(-0.17 \text { to }-0.04)^{* *}$ & $-0.11(-0.20 \text { to }-0.02)^{*}$ & $0.00(-0.00$ to 0.00$)$ & $-0.01(-0.03$ to 0.00$)$ & $-3.23(-4.91 \text { to }-1.55)^{* *}$ \\
\hline No family stress & Reference & Reference & Reference & Reference & Reference \\
\hline Family stress & $0.93(-0.87$ to 2.74$)$ & $0.67(-1.77$ to 3.12$)$ & $0.00(-0.01$ to 0.01$)$ & $0.33(-0.13$ to 0.79$)$ & $39.14(-3.54$ to 81.83$)$ \\
\hline GA & $-58.93(-67.28 \text { to }-50.58)^{* * *}$ & $-33.31(-44.73 \text { to }-21.90)^{* * *}$ & $0.08(0.04-0.11)^{* * *}$ & $3.35(3.28-3.42)^{* * *}$ & $-752.2(-763.5 \text { to }-740.3)^{* * *}$ \\
\hline $\mathrm{GA}^{2}$ & $7.13(6.34-7.92)^{* * *}$ & $4.30(3.21-5.38)^{* * *}$ & - & - & - \\
\hline $\mathrm{GA}^{3}$ & - & - & - & $-0.00(-0.00 \text { to }-0.00)^{* * * *}$ & - \\
\hline $\mathrm{GA}^{-0.5}$ & - & - & $-31.30(-47.83 \text { to }-14.77)^{* * *}$ & - & - \\
\hline $\ln (\mathrm{GA})$ & - & - & $-4.93(-7.43 \text { to }-2.42)^{* * *}$ & - & - \\
\hline $\mathrm{GA} \times \ln (\mathrm{GA})$ & - & - & - & - & $207.2(204.6-209.7)^{* * *}$ \\
\hline $\mathrm{GA}^{2} \times \ln (\mathrm{GA})$ & $-1.54(-1.71 \text { to }-1.37)^{* * *}$ & $-0.92(-1.15 \text { to }-0.69)^{* * *}$ & - & - & - \\
\hline GA $\times$ no family stress & Reference & Reference & Reference & Reference & Reference \\
\hline $\mathrm{GA} \times$ family stress & $-0.06(-0.14$ to 0.01$)$ & $-0.03(-0.14$ to 0.07$)$ & $0.00(-0.00$ to 0.00$)$ & $-0.02(-0.03$ to 0.00$)$ & $-1.78(-3.70$ to 0.13$)$ \\
\hline
\end{tabular}

\section{GA, Gestational age.}

Values are given as $\beta$ coefficient (95\% confidence interval).

${ }^{a}$ Models were constructed using fractional polynomials for gestational age and adjusted for fetal sex, maternal age, height, body mass index, education, ethnicity, smoking during pregnancy, parity, gestational diabetes, hypertension in pregnancy and pre-eclampsia.

${ }^{\mathrm{b}} \beta$ Coefficients are relative to the respective group of no maternal distress during pregnancy.

${ }^{*} p<0.05,{ }^{* *} p<0.01, * * * p<0.001$. 


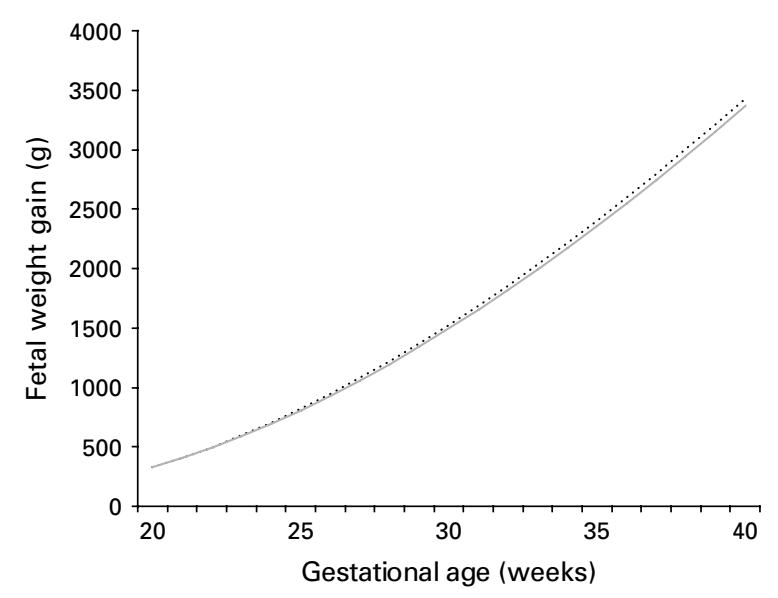

Fig. 1. Maternal anxious symptoms during pregnancy and fetal weight gain. Values are weight gain patterns of fetuses of mothers with (-) and without (…...) anxious symptoms during pregnancy based on linear mixed models that were adjusted for gestational age, fetal sex, maternal age, height, body mass index, education, ethnicity, smoking during pregnancy, parity, gestational diabetes, hypertension in pregnancy and pre-eclampsia.

organs more than that of distal body parts and bone structure.

Family stress was not related to fetal growth independently of maternal anxious symptoms. Arguably, we over-corrected our analyses by also adjusting for anxious symptoms. It is possible that aspects of family stress, such as lack of trust in family members, is one cause of anxious symptoms in pregnant women.

Our findings support the notion that maternal distress affects fetal head growth. As head circumference correlates with brain volume (Cooke et al. 1977), fetal head growth can be interpreted as an indicator of fetal brain development. Earlier studies reported a relation of maternal distress in pregnancy with childhood behavioural problems and poorer growth in infancy (O'Connor et al. 2002; Rahman et al. 2004). Moreover, previous research showed that intrauterine growth restriction indexed by birth length is associated with childhood behavioural problems and that head circumference at birth predicts cognitive functioning in childhood (Gale et al. 2006; Wiles et al. 2006). Possibly, fetal head growth is an intermediate in the relation of maternal psychological distress during pregnancy and subsequent child development.

Whereas our results showed that maternal distress is negatively related to several indicators of growth we observed no association with asymmetric fetal growth. This suggests that maternal distress during pregnancy leads to generally reduced fetal growth patterns but not to asymmetric growth restriction. Furthermore, these findings imply that the fetal brain is not spared when the fetus is exposed to maternal psychological distress.

Several mechanisms have been put forward to explain the association between maternal distress in pregnancy and fetal growth. Human and animal research suggests that maternal stress and distress during pregnancy leads to an elevated maternal hypothalamus-pituitary-adrenal (HPA) axis activity, which causes an increased release of glucocorticoids (Huizink et al. 2004; Mancuso et al. 2004; Diego et al. 2006), that in turn negatively affect fetal development (Mancuso et al. 2004; Diego et al. 2006). Maternal stress hormones may be transduced to the fetus by transplacental transport and by stress-induced release of placental hormones that enter the fetal circulation (Huizink et al. 2004). It was shown that maternal cortisol levels are strongly correlated with fetal levels, although fetal concentrations are lower compared with maternal concentrations (Gitau et al. 1998). Glucocorticoids are involved in fetal tissue proliferation and differentiation and are growth inhibiting (Fowden \& Forhead, 2004; Huizink et al. 2004). It is also possible that the association between maternal distress and fetal growth might be partly accounted for by a general reduced food intake of the mother or by a low intake of essential fatty acids or vitamins, such as folic acid or vitamin $\mathrm{B}_{12}$.

Our results might also be explained by an underlying common genetic factor affecting both maternal distress and fetal growth. Although we controlled for genetic effects on fetal growth by adjusting for maternal height and pre-pregnancy body mass index, residual genetic influences are likely.

The main strength of this large prospective population-based cohort study was that the repeated fetal ultrasound assessments were combined with information on birth weight, so that we were able to assess fetal growth from mid-pregnancy until birth. In addition, we controlled for many confounders known to affect fetal development.

Several potential limitations must be considered. As maternal psychological distress was only assessed at 20 weeks of pregnancy, we do not know whether maternal affective symptoms and family stress varied in intensity or were persistent throughout pregnancy. Second, the anxiety and depression scale of the BSI were strongly correlated. It seems plausible that these scales measure very similar concepts. This reflects the co-morbidity between anxiety and depression, which has frequently been reported (Beekman et al. 2000). We could not disentangle whether maternal anxiety and depression have independent effects on fetal growth, because of collinearity and possible overadjustment in our analysis. Moreover, we were not able to control for antidepressant drug use during 
pregnancy. A recent population-based study $(n=$ 29005) of Dutch pregnant women showed, however, that only $1.8-2 \%$ took antidepressants at some point during pregnancy (Ververs et al. 2006). Our data also do not allow us to determine which physiological mechanisms may account for the findings of this study. As data on maternal distress were more complete in Dutch and higher-educated mothers whose children had a higher birth weight, we cannot rule out selection effects on fetal growth trajectories. Finally, while the size of the association between maternal psychological distress and fetal growth was small, such effects may be important in public health terms. The relations between maternal psychological distress and outcomes were evident within the normal range of maternal distress and fetal growth. Possibly the observed effects would have been larger if more individuals with higher rates of maternal distress and lower rates of fetal growth had been studied.

In conclusion, maternal psychological distress during pregnancy affects fetal development. Future research should address mechanisms underlying the relation between maternal psychological distress and fetal growth, e.g. dysregulation of the HPA axis, and long-term effects on child development. Furthermore, our findings highlight the importance of distress in pregnant women because this may affect the fetus. Information about distress can easily be obtained by questionnaires. Pregnant women at elevated risk could then be invited to participate, for example, in stress-reduction programmes.

\section{Acknowledgements}

The Generation R Study is conducted by the Erasmus Medical Centre (Rotterdam, The Netherlands) in close collaboration with the School of Law and Faculty of Social Sciences of the Erasmus University Rotterdam, the Municipal Health Service Rotterdam area, the Rotterdam Homecare Foundation and the Stichting Trombosedienst \& Artsenlaboratorium Rijnmond (STAR), Rotterdam. We gratefully acknowledge the contribution of participating mothers and their children, general practitioners, hospitals, midwives and pharmacies in Rotterdam. The first phase of the Generation R Study was made possible by financial support from the Erasmus Medical Centre, Rotterdam, the Erasmus University Rotterdam and the Netherlands Organization for Health Research and Development (Zon Mw).

\section{Declaration of Interest}

None.

\section{References}

Altman DG, Bland JM (1994). Diagnostic tests 2: Predictive values. British Medical Journal 309, 102.

Altman DG, Chitty LS (1997). New charts for ultrasound dating of pregnancy. Ultrasound in Obstetrics and Gynecology 10, 174-191.

Andersson L, Sundstrom-Poromaa I, Wulff M, Astrom M, Bixo M (2004). Neonatal outcome following maternal antenatal depression and anxiety: a population-based study. American Journal of Epidemiology 159, 872-881.

Andrews G, Peters L (1998). The psychometric properties of the Composite International Diagnostic Interview. Social Psychiatry and Psychiatric Epidemiology 33, 80-88.

Beekman AT, de Beurs E, van Balkom AJ, Deeg DJ, van Dyck R, van Tilburg W (2000). Anxiety and depression in later life: co-occurrence and communality of risk factors. American Journal of Psychiatry 157, 89-95.

Birtchnell J, Evans C, Kennard J (1988). The total score of the Crown-Crisp Experiential Index: a useful and valid measure of psychoneurotic pathology. British Journal of Medical Psychology 61, 255-266.

Bloomfield FH, Oliver MH, Harding JE (2006). The late effects of fetal growth patterns. Archives of Disease in Childhood. Fetal and Neonatal Edition 91, F299-F304.

Byles J, Byrne C, Boyle MH, Offord DR (1988). Ontario Child Health Study - reliability and validity of the general functioning subscale of the McMaster Family Assessment Device. Family Process 27, 97-104.

Cooke RW, Lucas A, Yudkin PL, Pryse-Davies J (1977). Head circumference as an index of brain weight in the fetus and newborn. Early Human Development 1, 145-149.

De Beurs E (2004). Brief Symptom Inventory, Handleiding (Manual). Pits Publishers: Leiden, The Netherlands.

Diego MA, Jones NA, Field T, Hernandez-Reif M, Schanberg S, Kuhn C, Gonzalez-Garcia A (2006). Maternal psychological distress, prenatal cortisol, and fetal weight. Psychosomatic Medicine 68, 747-753.

Evans J, Heron J, Patel RR, Wiles N (2007). Depressive symptoms during pregnancy and low birth weight at term: longitudinal study. British Journal of Psychiatry 191, $84-85$.

Ferreira AJ (1965). Emotional factors in prenatal environment. A review. Journal of Nervous and Mental Disease 141, 108-118.

Fowden AL, Forhead AJ (2004). Endocrine mechanisms of intrauterine programming. Reproduction 127, 515-526.

Gale CR, O'Callaghan FJ, Bredow M, Martyn CN, Avon Longitudinal Study of Parents and Children Study Team (2006). The influence of head growth in fetal life, infancy, and childhood on intelligence at the ages of 4 and 8 years. Pediatrics 118, 1486-1492.

Gitau R, Cameron A, Fisk NM, Glover V (1998). Fetal exposure to maternal cortisol. Lancet 352, 707-708.

Hadlock FP, Harrist RB, Carpenter RJ, Deter RL, Park SK (1984). Sonographic estimation of fetal weight. The value of femur length in addition to head and abdomen measurements. Radiology 150, 535-540.

Hanson M (2002). Birth weight and the fetal origins of adult disease. Pediatric Research 52, 473-474. 
Hedegaard M, Henriksen TB, Sabroe S, Secher NJ (1993). Psychological distress in pregnancy and preterm delivery. British Medical Journal 307, 234-239.

Huizink AC, Mulder EJ, Buitelaar JK (2004). Prenatal stress and risk for psychopathology: specific effects or induction of general susceptibility? Psychological Bulletin 130, 115-142.

Jaddoe VW, Mackenbach JP, Moll HA, Steegers EA, Tiemeier H, Verhulst FC, Witteman JC, Hofman A (2006). The Generation R Study: design and cohort profile. European Journal of Epidemiology 21, 475-484.

Kramer MS (1987). Determinants of low birth weight: methodological assessment and meta-analysis. Bulletin of the World Health Organization 65, 663-737.

Kurki T, Hiilesmaa V, Raitasalo R, Mattila H, Ylikorkala O (2000). Depression and anxiety in early pregnancy and risk for preeclampsia. Obstetrics and Gynecology 95, 487-490.

Lesage J, Del-Favero F, Leonhardt M, Louvart H, Maccari S, Vieau D, Darnaudery M (2004). Prenatal stress induces intrauterine growth restriction and programmes glucose intolerance and feeding behaviour disturbances in the aged rat. Journal of Endocrinology 181, 291-296.

Lou HC, Hansen D, Nordentoft M, Pryds O, Jensen F, Nim J, Hemmingsen R (1994). Prenatal stressors of human life affect fetal brain development. Developmental Medicine and Child Neurology 36, 826-832.

Mancuso RA, Schetter CD, Rini CM, Roesch SC, Hobel CJ (2004). Maternal prenatal anxiety and corticotropinreleasing hormone associated with timing of delivery. Psychosomatic Medicine 66, 762-769.

Nakano Y, Oshima M, Sugiura-Ogasawara M, Aoki K, Kitamura T, Furukawa TA (2004). Psychosocial predictors of successful delivery after unexplained recurrent spontaneous abortions: a cohort study. Acta Psychiatrica Scandinavica 109, 440-446.

Nordentoft M, Lou HC, Hansen D, Nim J, Pryds O, Rubin P, Hemmingsen R (1996). Intrauterine growth retardation and premature delivery: the influence of maternal smoking and psychosocial factors. American Journal of Public Health 86, 347-354.

O'Connor TG, Heron J, Glover V, Alspac Study Team (2002). Antenatal anxiety predicts child behavioral/ emotional problems independently of postnatal depression. Journal of the American Academy of Child and Adolescent Psychiatry 41, 1470-1477.

Pinto ML, Shetty PS (1995). Influence of exercise-induced maternal stress on fetal outcome in Wistar rats: inter-generational effects. British Journal of Nutrition 73, 645-653.

Rahman A, Bunn J, Lovel H, Creed F (2007). Association between antenatal depression and low birthweight in a developing country. Acta Psychiatrica Scandinavica 115, 481-486.

Rahman A, Iqbal Z, Bunn J, Lovel H, Harrington R (2004). Impact of maternal depression on infant nutritional status and illness : a cohort study. Archives of General Psychiatry 61, 946-952.

Rondo PH, Ferreira RF, Nogueira F, Ribeiro MC, Lobert H, Artes R (2003). Maternal psychological stress and distress as predictors of low birth weight, prematurity and intrauterine growth retardation. European Journal of Clinical Nutrition 57, 266-272.

Royston P, Altman DG (1994). Regression using fractional polynomials of continuous covariates: parsimonious parametric modelling (with discussion). Applied Statistics 43, 429-467.

Royston P, Ambler G, Sauerbrei W (1999). The use of fractional polynomials to model continuous risk variables in epidemiology. International Journal of Epidemiology 28, 964-974.

Roza SJ, Verburg BO, Jaddoe VW, Hofman A, Mackenbach JP, Steegers EA, Witteman JC, Verhulst FC, Tiemeier H (2007). Effects of maternal smoking in pregnancy on prenatal brain development. The Generation R Study. European Journal of Neuroscience 25, 611-617.

Verburg BO, Mulder PG, Hofman A, Jaddoe VW, Witteman JC, Steegers EA (2008a). Intra- and interobserver reproducibility study of early fetal growth parameters. Prenatal Diagnosis 28, 323-331.

Verburg BO, Steegers EA, De Ridder M, Snijders RJ, Smith E, Hofman A, Moll HA, Jaddoe VW, Witteman JC (2008b). New charts for ultrasound dating of pregnancy and assessment of fetal growth: longitudinal data from a population-based cohort study. Ultrasound in Obstetrics and Gynecology 31, 388-396.

Ververs T, Kaasenbrood H, Visser G, Schobben F, de Jong-van den Berg L, Egberts T (2006). Prevalence and patterns of antidepressant drug use during pregnancy. European Journal of Clinical Pharmacology 62, 863-870.

Wiles NJ, Peters TJ, Heron J, Gunnell D, Emond A, Lewis G (2006). Fetal growth and childhood behavioral problems: results from the ALSPAC cohort. American Journal of Epidemiology 163, 829-837. 